\title{
Short term effects of aerobic training in the clinical management of moderate to severe asthma in children
}

\author{
J Alberto Neder, Luiz E Nery, Antônio C Silva, Anna L B Cabral, Ana L G Fernandes
}

Respiratory Division, Department of Medicine

J A Neder

L E Nery

A L G Fernandes

Department of Physiology A C Silva

Federal University of Sao Paulo-Paulista School of Medicine (UNIFESP-EPM), Sao Paulo, Brazil

Center of Sport Practices of the University of Sao Paulo (CEPEUSP), Sao Paulo, Brazil A L B Cabral

Correspondence to: Dr J A Neder, Department of Physiology, St George's Hospital Medical School, Cranmer Terrace, London SW17 ORE, UK.

Received 11 December 1997 Returned to authors 27 February 1998 Revised version received 23 March 1998

Accepted for publication

3 June 1998

\begin{abstract}
Background-Aerobic training has a number of well known beneficial effects in both normal and asthmatic children. However, the impact of training on the clinical management of the underlying bronchial asthma remains controversial, particularly in the most severe patients.

Methods-Clinical evaluation, spirometric tests, symptom limited maximum exercise testing, and exercise challenge tests were performed in a group of children with stable moderate to severe asthma. Forty two patients ( 24 boys) aged 8-16 were evaluated twice: before and after supervised aerobic training (group 1, $n=26$ ) and two months apart (untrained group $2, \mathbf{n}=16$ ).

Results-Spirometric and maximal exercise variables in the initial evaluation were significantly reduced in group $1(p<0.05)$ but medication and clinical scores and the occurrence of exercise induced bronchospasm (EIB) did not differ between the two groups. Aerobic improvement with training (maximal oxygen uptake and/or anaerobic threshold increment $>10 \%$ and $100 \mathrm{ml}$ ) was inversely related to the baseline level of fitness and was independent of disease severity. Although the clinical score and the occurrence of EIB did not change after training, aerobic improvement was associated with a significant reduction in the medication score and the daily use of both inhaled and oral steroids $(\mathbf{p}<0.05)$.

Conclusions-Aerobic improvement with training in less fit asthmatic children is related to a short term decrease in the daily use of inhaled and oral steroids, independent of the severity of the disease. (Thorax 1999;54:202-206)
\end{abstract}

Keywords: asthma; children; exercise training; maximal oxygen uptake; anaerobic threshold; physical fitness

Regular physical activity of adequate intensity and duration involving large muscle groups has been proved to have a number of potential beneficial effects on general health, including improvement in aerobic capacity, body composition, flexibility, muscular strength, and psychosocial measures. ${ }^{1}$ This improvement might be particularly true for patients who suffer from chronic diseases such as congestive heart failure, chronic obstructive pulmonary disease (COPD), and bronchial asthma. However, patients with chronic respiratory diseases tend to show less tolerance to exercise due to pulmonary limitation, self-restriction of activities, or lack of physical activity secondary to medical advice or family influence. ${ }^{2}$ Thus, children with bronchial asthma, primarily those with a clinically more severe disease, tend to have a sedentary lifestyle and therefore be inclined to have lower aerobic fitness than their healthy non-asthmatic peers. ${ }^{2-7}$

However, most of the published studies have failed to show a clinical advantage of the improvement in fitness with training. ${ }^{2568}$ Ludwick et al found that normalisation of cardiopulmonary endurance in 65 severe asthmatic children was not related to improvement in pulmonary function. ${ }^{5}$ Robinson et al showed that significant aerobic improvement with training was not associated with changes in use of medication, symptoms, or bronchial responsiveness to histamine. ${ }^{6}$ Cochrane and Clark reported that fitness level was not associated with several clinical and functional characteristics of the disease. ${ }^{3}$ Recently, Thio et al found that normalisation of cardiovascular fitness in asthmatic children was not related to a lower prevalence of exercise induced bronchospasm (EIB). ${ }^{8}$ These results contrast with the traditional notion that aerobic improvement may have a number of theoretical advantages for asthma control. ${ }^{2}$ The objective of this study was to analyse the association between possible changes in aerobic capacity after training and the clinical and functional markers of the severity of bronchial asthma in a group of children with moderate to severe disease.

\section{Methods}

PATIENTS

Forty two asthmatic children (24 boys) of mean (SD) age 12.4 (1.8) years (range 8-16) were studied in Sao Paulo, Brazil. The mean (SD) body mass and height were $40.4(9.8) \mathrm{kg}$ and $146.8(10.3) \mathrm{cm}$, respectively. Although they were physically active, none was following a regular exercise training programme. Medical follow up was performed by the same staff physician. The children were in a stable phase of the disease, with no exacerbation during the 15 days before the start of the tests. The study was approved by the appropriate institutional medical ethics committee and written informed consent was obtained from the patients and their parents. No remuneration was offered.

The subjects were referred from an asthma management centre and were consecutively enrolled in an aerobic training programme. 
Due to technical and operational limitations the number of patients trained was restricted to the first 26 children (group 1, 15 boys and 11 girls). The remaining 16 subjects had no training (group 2, 9 boys and 7 girls).

STUDY PROTOCOL

A clinical and medication history was obtained from all subjects, followed by physical examination, spirometric tests before and after bronchodilator, and a progressive incremental cardiopulmonary exercise test on a cycle ergometer. On another day the patients performed an exercise challenge test. This protocol was performed twice, before and after training by patients in group 1 and two months apart by those in group 2 .

\section{MEASUREMENTS}

Clinical evaluation

According to the clinical parameters of the International Consensus Report on Diagnosis and Management of Asthma, ${ }^{9}$ severe asthma in children is defined as frequent bronchospasm and nocturnal asthmatic symptoms almost daily, peak expiratory flow (PEF) values $<60 \%$ predicted at baseline despite optimal therapy, daily use of inhaled anti-inflammatory agents at high doses (beclomethasone $>800 \mu \mathrm{g} /$ day), and frequent use of systemic corticosteroids ( $>3$ times/year). Moderate asthma is defined as symptoms requiring inhaled $\beta_{2}$ agonists almost daily and nocturnal asthmatic symptoms more than twice a month but not daily, PEF values of $60-80 \%$ predicted at baseline but normal after bronchodilator, and daily use of an antiinflammatory agent at low or moderate doses (beclomethasone $<800 \mu \mathrm{g} /$ day).

All patients were on an inhaled corticosteroid (beclomethasone $500-1500 \mu \mathrm{g} /$ day) and $33 \mathrm{had}$ a past history of frequent courses of oral corticosteroids ( $>3$ times/year); nine patients were on systemic steroids at the time of the study. According to the guidelines for the evaluation of impairment/disability in patients with asthma, ${ }^{10} 33$ patients had a medication score of $>3$ requiring treatment for adequate clinical control: bronchodilator on demand, inhaled beclomethasone in a daily dose of $\geqslant 800 \mu \mathrm{g}$, or more than three courses of oral steroids per year.

SPIROMETRIC TESTS

Patients were asked to refrain from short acting bronchodilators for at least six hours before testing. Spirometric tests were performed in all subjects before and 10 minutes after the inhalation of $200 \mu \mathrm{g}$ salbutamol via a pressurised metered dose inhaler connected to a spacer. The equipment used was a CPF-S (Medical Graphics Corporation, St Paul, Minnesota, USA) with flow measurement carried out with a pneumotachograph Fleisch No. 3. Technical procedures, acceptability and reproducibility criteria were those recommended by the American Thoracic Society. ${ }^{11}$ Predicted normal values for all spirometric variables were those of Knudson et al..$^{12}$ A positive response to bronchodilator was defined as a $12 \%$ and
$200 \mathrm{ml}$ increase in the forced expiratory volume in one second $\left(\mathrm{FEV}_{1}\right){ }^{11}$

CARDIOPULMONARY EXERCISE TESTING

Exercise tests were performed using a digital computer based exercise system (MGC-CPX System; Medical Graphics Corporation (MGC), St Paul, Minnesota, USA) with breath by breath analysis of metabolic, ventilatory, and cardiovascular variables. The maximal symptom limited exercise test was carried out on a calibrated electromagnetically braked cycle ergometer (CPE 2000; Medical Graphics Corporation), modified with child pedal cranks. The selected work rate was continuously increased in a linear ramp pattern $(15 \mathrm{~W} / \mathrm{min}$ for height $<150 \mathrm{~cm} ; 20 \mathrm{~W} / \mathrm{min}$ for height $>150 \mathrm{~cm})^{13}$ so that the duration of the incremental exercise test was more than eight and less than 12 minutes. The following data were recorded as a moving average of eight breaths: oxygen uptake $\left(\dot{\mathrm{V}}_{2}, \mathrm{ml} / \mathrm{min} \mathrm{STPD}\right)$; carbon dioxide production $\left(\dot{\mathrm{V}}_{\mathrm{CO}_{2}}, \mathrm{ml} / \mathrm{min}\right.$ STPD); respiratory exchange ratio (R); minute ventilation ( $\dot{\mathrm{V}} \mathrm{E}, 1 / \mathrm{min}$ BTPS), respiratory rate (f, bpm); ventilatory equivalent for oxygen and carbon dioxide $\left(\dot{\mathrm{V}} \mathrm{E} / \dot{\mathrm{V}}_{2}\right.$ and $\left.\dot{\mathrm{V}} \mathrm{E} / \dot{\mathrm{V}}_{\mathrm{CO}}\right)$; end tidal partial pressures of oxygen and carbon dioxide $\left(\mathrm{PETO}_{2}\right.$ and $\left.\mathrm{PeTCO}_{2}, \mathrm{~mm} \mathrm{Hg}\right)$; heart rate $(\mathrm{HR}, \mathrm{bpm})$, and oxygen pulse $\left(\dot{\mathrm{V}}_{2} / \mathrm{HR}\right.$, $\mathrm{ml} /$ beat).

The predicted $\dot{\mathrm{V}}_{2}$ max was calculated according to the equations of Cooper et al for children, ${ }^{14}$ taking into account the close demographic and anthropometric similarity of the subjects. The lower limit of normality for $\dot{\mathrm{V}}_{2} \max$ was defined according to the values proposed by these authors (lower 95\% confidence limit; estimated $-1.64 \times \mathrm{SD}$ ): girls $<11$ years $=25 \mathrm{ml} / \mathrm{min} \cdot \mathrm{kg}$; girls $>11$ years $=27 \mathrm{ml} /$ min $\cdot \mathrm{kg}$; boys $<13$ years $=32 \mathrm{ml} / \mathrm{min} \cdot \mathrm{kg}$; boys $>13$ years $=37 \mathrm{ml} / \mathrm{min} \bullet \mathrm{kg}$. The $\dot{\mathrm{V}}_{2}$ at the anaerobic threshold $\left(\dot{\mathrm{V}}_{2} \mathrm{AT}\right)$ was measured by the gas exchange method, visually checking the

Table 1 Clinical and functional characteristics of the asthmatic children at baseline and final evaluations

\begin{tabular}{|c|c|c|c|}
\hline Variables & & $\begin{array}{l}\text { Group 1 } \\
(n=26)\end{array}$ & $\begin{array}{l}\text { Group 2 } \\
(n=16)\end{array}$ \\
\hline \multirow{2}{*}{$\begin{array}{l}\mathrm{FEV}_{1} \text { pre-BD (\% } \\
\text { pred) }\end{array}$} & In & $78.3(16.4)$ & $99.1(18.8)^{\star}$ \\
\hline & & .5) & $97.6(17.4)^{\star}$ \\
\hline \multirow{2}{*}{$\begin{array}{l}\mathrm{FEV}_{1} \text { post-BD (\% } \\
\text { pred) }\end{array}$} & Initial & $92.1(18.7)$ & $109.0(19.3)^{\star}$ \\
\hline & & $91.4(17.1)$ & $106.5(15.3)^{\star}$ \\
\hline \multirow{2}{*}{$\dot{\mathrm{V}}_{2} \max (\%$ pred $)$} & Initial & $83.4(10.5)$ & $99.0(19.4)^{\star}$ \\
\hline & Final & $87.8(1$ & $91.1(18.0)$ \\
\hline \multirow[t]{2}{*}{$\dot{\mathrm{V}}_{2} \max (1 / \mathrm{min})$} & Initial & $1444(215)$ & $1659(287)^{\star}$ \\
\hline & & & \\
\hline \multirow{2}{*}{$\dot{\mathrm{V}} \mathrm{O}_{2} \mathrm{AT} / \dot{\mathrm{V}} \mathrm{O}_{2} \max (\%)$} & Initial & 46.2 & $59.5(12.6)^{\star}$ \\
\hline & Final & $48.3(9.1)$ & $47.2(13.4) \dagger$ \\
\hline \multirow[t]{2}{*}{$\mathrm{O}_{2}$ pulse (\% pred) } & Initial & $91.2(14.1)$ & $114.1(23.3)^{\star}$ \\
\hline & Final & $106.5(17.1) \dagger$ & $106.3(30.1)$ \\
\hline \multirow{2}{*}{ Severe asthma ${ }^{9}$} & Initial & $22(84 \%)$ & $11(69 \%)$ \\
\hline & Final & $18(69 \%)$ & $13(81 \%)$ \\
\hline \multirow[t]{2}{*}{ Medication score $>3^{10}$} & Initial & $22(84 \%)$ & $11(69 \%)$ \\
\hline & Final & $15(57 \%)$ & $14(87 \%)$ \\
\hline \multirow[t]{2}{*}{ EIB positive ${ }^{16}$} & Initial & & \\
\hline & Final & $12(46 \%)$ & $10(71 \%)$ \\
\hline
\end{tabular}

Values are mean (SD) for continuous variables and frequency (approximated \%) for the others.

$\mathrm{FEV}_{1}=$ forced expiratory volume in one second; $\mathrm{FVC}=$ forced vital capacity; $\mathrm{BD}=$ bronchodilator; $\dot{\mathrm{Vo}}_{2}=$ oxygen consumption; $\mathrm{AT}=$ anaerobic threshold; $\mathrm{O}_{2}$ pulse $=\dot{\mathrm{V}}_{2} /$ heart rate; $\max$ $=$ relative to maximum exercise.

${ }^{\star} \mathrm{p}<0.05$ (group $2>$ group 1 ; non-paired $t$ test)

tp $<0.05$ (final evaluation vs initial evaluation; paired $t$ test). 


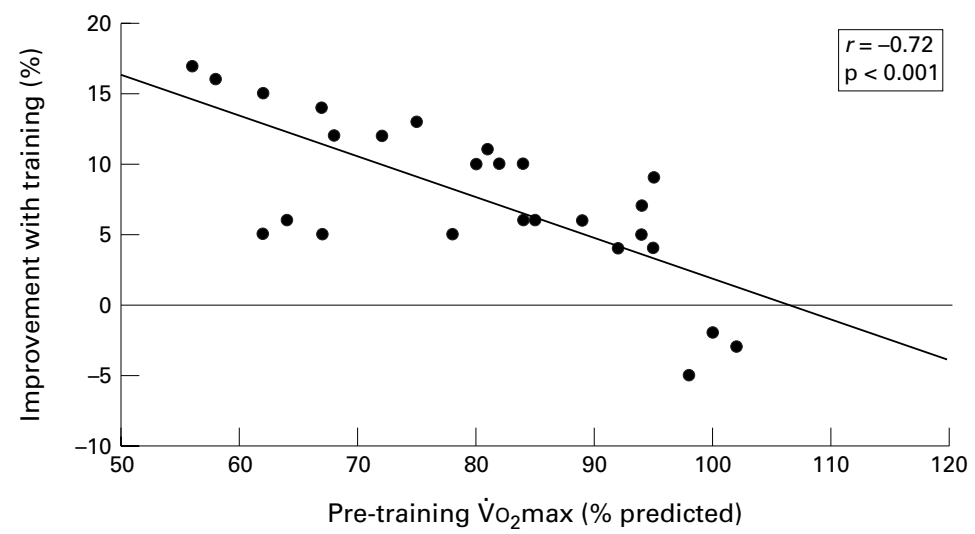

Figure 1 Relationship between baseline maximal aerobic fitness and degree of improvement after training in 26 children with moderate to severe asthma.

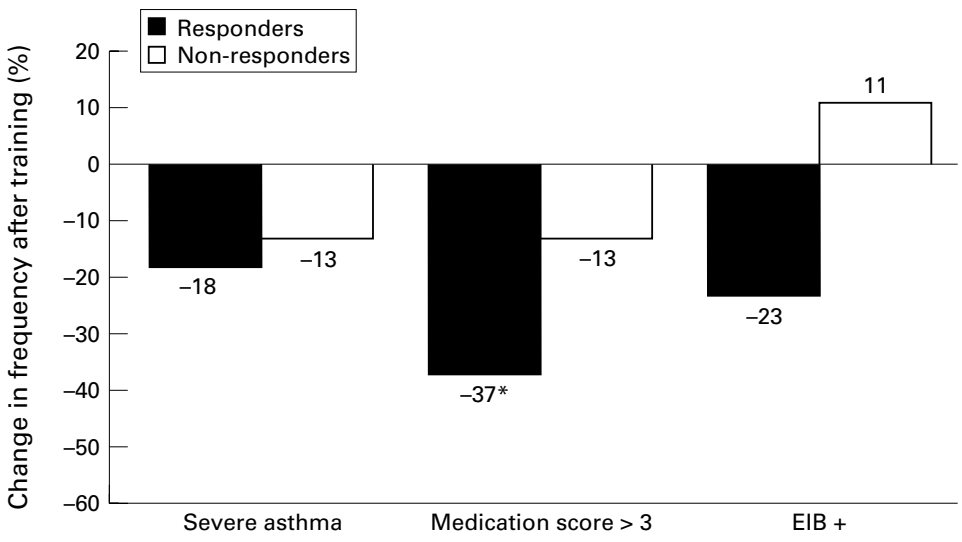

Figure 2 Association between changes in clinical indicators of asthma severity and positive (responders) or negative (non-responders) response to aerobic training. ${ }^{\star} p<0.05$ (Fisher's exact test).

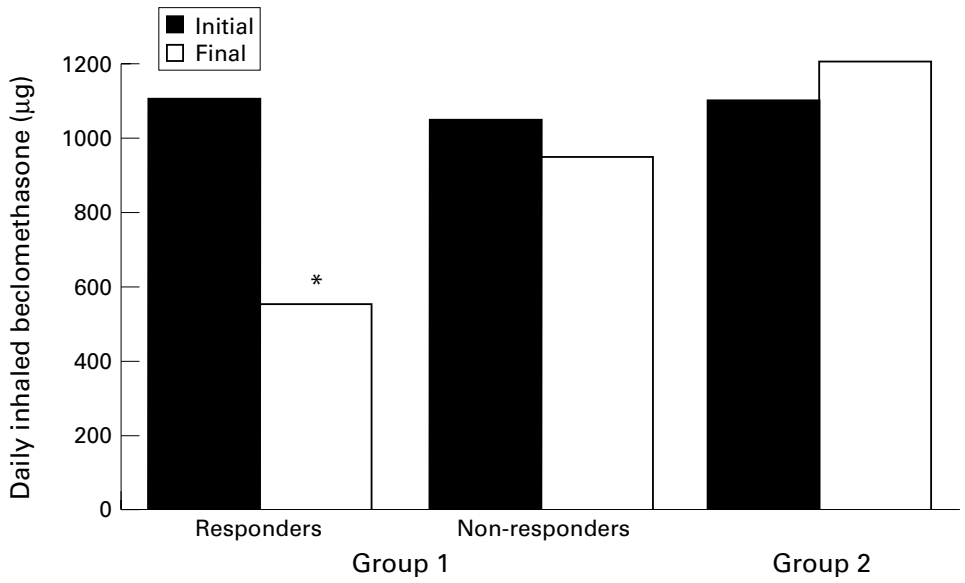

Figure 3 Mean values of daily inhaled beclomethasone in the initial and final evaluations. Group 1 = trained children with (responders) and without (non-responders) aerobic improvement after training; group $2=$ untrained children. ${ }^{*} p<0.05$ (paired $t$ test).

inflection point of $\dot{\mathrm{V}} \mathrm{CO}_{2}$ with regard to $\dot{\mathrm{V}}_{2}$ (modified $\mathrm{V}$ slope), ${ }^{15}$ and by the ventilatory method in which $\dot{\mathrm{VE}} / \dot{\mathrm{V}}_{2}$ and $\mathrm{PETO}_{2}$ increased while $\dot{\mathrm{V}} / \dot{\mathrm{V}}_{\mathrm{CO}}$ and $\mathrm{PETCO}_{2}$ remained stable. The lower limit of normality for $\dot{\mathrm{V}}_{2} \mathrm{AT}$ was defined as $40 \%$ of the $\dot{\mathrm{V}}_{2}$ max predicted since, of the 109 children evaluated by Cooper et al, only one presented with a value below this cut off point. ${ }^{14}$

EXERCISE CHALLENGE TEST

The test for detection of EIB was carried out with a mechanically braked Monark $^{\circledR}$ cycle ergometer. Methylxanthines and $\beta_{2}$ adrenoceptor agonists were withheld 12 hours prior to the test. Before the exercise the patient performed a forced expiratory manoeuvre (Vitatrace ${ }^{\circledR}$ spirometer) with assessment of basal values of forced vital capacity (FVC) and $\mathrm{FEV}_{1}$. The test was carried out only in patients with normal spirometric values at rest - that is, $\mathrm{FEV}_{1}$ above the lower $95 \%$ confidence limit $(n=35) .{ }^{12}$ The tests were performed at a room temperature of $18-26^{\circ} \mathrm{C}$, barometric pressure $680-$ $703 \mathrm{~mm} \mathrm{Hg}$, and relative humidity $55-60 \%$. After one minute of light exercise on the cycle ergometer the work load was quickly increased until the heart rate corresponded to $80 \%$ of predicted (220-age), and then maintained for six minutes. ${ }^{16}$ Spirometric evaluations were performed successively at five, 10, and 20 minutes after the exercise. EIB was defined as being present if $\mathrm{FEV}_{1}$ showed a reduction equal to or greater than $10 \%$ of its pre-exercise values. ${ }^{16}$

EXERCISE TRAINING

The two month indoor aerobic training programme on a cycle ergometer was performed by 26 asthmatic children (group 1). Medical supervision was provided during all training sessions. The sessions were performed three times a week (total 24). Inhaled $\beta_{2}$ adrenergic bronchodilators were administered 10 minutes before training. The target heart rate during the training sessions was individualised. This initially corresponded to the anaerobic threshold and was increased every two weeks to an intensity which the subject was able to endure for 30 minutes. A typical session consisted of 10-15 minutes of callisthenics and stretching warm up exercises, 30 minutes of continuous aerobic activity on a cycle ergometer, and a five minute period of cooling down.

STATISTICAL ANALYSIS

Mean (SD) values are reported. Paired and non-paired $t$ tests were used to compare within group and between group evaluations, respectively; the $\chi^{2}$ (Fisher exact) test to evaluate the association between changes in clinical and functional outcomes and response to training; and Pearson product moment correlation to assess linear association between variables. The level of statistical significance was always set at $\mathrm{p}<0.05$.

\section{Results}

INITIAL EVALUATION

In the basal evaluation mean (SD) values of the main spirometric variables were significantly lower in patients in group 1 than in those in group 2 (table 1). However, as anticipated by clinical stability, only two patients in group 1 had post-bronchodilator $\mathrm{FEV}_{1}$ values below the lower $95 \%$ confidence limit. Furthermore, in patients in group 1 the main parameters of maximum aerobic performance $\left(\dot{\mathrm{V}}_{2} \mathrm{max}\right.$, $\dot{\mathrm{V}}_{2} \mathrm{AT}, \mathrm{O}_{2}$ pulse max) were significantly reduced when compared with those of group 2 . On the other hand, the clinical and medication scores and prevalence of a positive EIB test were not significantly different between the two groups. 
FINAL EVALUATION

The supervised aerobic training was well tolerated and completed by all children in group 1 . Only one child experienced a mild episode of EIB during a training session, but it was promptly reversed with an inhaled bronchodilator. Although significant only for the $\mathrm{O}_{2}$ pulse $\max (\mathrm{p}<0.05)$, we found a trend towards an increase in the maximal exercise variables in the trained group. On the other hand, a worsening trend was identifiable in the untrained children, although this reached statistical significance only for $\dot{\mathrm{V}}_{2} \mathrm{AT}$. There were, however, no significant changes in the clinical outcomes in the two groups (table 1).

As the response to exercise training has consistently been reported to be inversely related to the baseline values, ${ }^{3}{ }^{17-19}$ we analysed the relationship between the degree of improvement (post-pre/pre) and the initial level of fitness. As shown in fig 1, there was a strongly negative relationship between these variablesthat is, the less fit the subject the higher the aerobic gain with training. A positive response to training $\left(\dot{\mathrm{V}}_{2} \max\right.$ and/or $\dot{\mathrm{V}}_{2} \mathrm{AT}$ post training-pre training/pre-training $\times 100$ more than $10 \%$ and $100 \mathrm{ml}$ ) was found in 11 subjects $(42 \%)$; all of these training "responders" presented an abnormally reduced $\dot{\mathrm{V}}_{2}$ max and $\dot{\mathrm{V}} \mathrm{O}_{2} \mathrm{AT}$ at the initial evaluation. Although clinical and functional variables at baseline did not differ between "responders" and "nonresponders", after training the "responders" had a lower prevalence of subjects with a medication score of $>3(p<0.05$, fig 2$)$. In addition, a significant reduction was seen in the mean dose of daily inhaled steroids in the "responders" group (from 1125 (225) to $575(150) \mu \mathrm{g} /$ day, $p<0.05$, fig 3). Finally, in five (of nine) children oral steroids were able to be withdrawn; interestingly, all of them showed aerobic improvement with training.

\section{Discussion}

We have evaluated the effect of a supervised aerobic training programme on the cardiorespiratory fitness and clinical indicators of control in a group of children with moderate to severe but stable asthma. The degree of response to training and the positive effect on the clinical management were strongly influenced by the level of fitness in the initial evaluation; beneficial effects were shown only in the less fit patients. Our results suggest that exercise therapy for the most untrained children can have a role, at least in the short term, in reducing the minimal medication needed for control of moderate to severe asthma.

Previous studies in normal and asthmatic patients have shown that the initial grade of fitness and motivation is an important predictor of aerobic improvement after training. ${ }^{37-19}$ Cochrane and Clark, for example, found that the relative gain in $\dot{\mathrm{V}}_{2}$ max after training in asthmatic subjects was negatively related to symptom score on the training day and the baseline level of fitness, and positively to motivation. ${ }^{3}$ Thus, the improvement after training in muscular capillarisation, oxidative capacity, muscular strength, and cardiocircula- tory adjustments is likely to occur in motivated subjects with worse baseline aerobic conditions. However, it should be recognised that the so called "regression to the mean" effect cannot be ruled out by this finding; the random longitudinal variation tends to increase the lower values of a given distribution or, alternatively, the higher values tend to randomly decrease with time. Nevertheless, as previously noted, this higher potential for improvement in the most unfit individuals is a well known phenomenon and it seems highly improbable that such a statistical artefact would be consistently related to another measurable biological effect such as the reduction in inhaled steroids (figs 2 and 3 ). In this context, there is now growing evidence to show that the systemic effects of inhaled beclomethasone in children are dose dependent ${ }^{2021}$ and medication usage seems to be a particularly useful index of overall asthma control. ${ }^{9}{ }^{10}$

Although the respiratory system is usually considered to be largely insensitive to training effects per se (with the possible exception of muscle ventilatory strength and endurance), a cause-effect relationship could explain this association. Thus, one can speculate that a possibly lower occurrence of EIB after training would induce a lower chronic release of inflammatory mediators and therefore reduce the need for inhaled steroids. However, we did not find a significant reduction in the prevalence of a positive EIB test with training, regardless of whether or not there was a positive response (fig 2). Another more plausible hypothesis is that the improvement was related to a higher degree of acceptance and level of self-care in the least fit patients who usually have negative attitudes toward their disease and exertion. ${ }^{22}{ }^{23}$ Thus, Strunk et $a l^{22}$ showed that the wide variability in aerobic performance in a group of 90 children with moderate and severe asthma was mainly related to the degree of social and disease adjustment. Engström $e t a l^{3}$ in a group of 10 severely asthmatic children submitted to physical training showed that only psychological modifications correlated significantly with aerobic improvement. Thus, individual variations in acceptance and knowledge of the disease seem to influence the usual level of physical activity in asthmatic children, and therefore their degree of fitness. In this context, exercise training may induce a more decided posture in relation to the disease, with consequences in the minimum medication required for clinical control.

Our results are consistent with those of Thio et $a l^{8}$ who were not able to find a lower prevalence of EIB after dynamic exercise training, although in a previous cross sectional study we found an association between a reduction in $\dot{\mathrm{V}}_{2} \mathrm{AT}$ and a higher prevalence of EIB in asthmatic children. ${ }^{24}$ While one can predict a reduction in EIB with aerobic improvement (secondary to training induced lower submaximal ventilation), ${ }^{25}$ in our study this enhancement alone was probably not sufficient to reduce the EIB, at least when assessed in a formal challenge test. 
A particularly notable finding was the relative inefficacy of the training programme in improving the maximal aerobic parameters in almost $60 \%$ of the children. However, one should recognise that maximal incremental testing is not representative of the daily pattern of exercise activities in the paediatric group (which is better characterised by short bursts of activity); new submaximal protocols have been suggested to be more suitable for evaluating training responses in children. ${ }^{26}$ In addition, the degree of fitness in the initial evaluation was above that expected for asthmatic children (table 1) and the low pre-intervention prevalence of unfit children could have induced a type II error. This finding is consistent, however, with the suggestion that secular trends do not reduce the average aerobic fitness of westernised children. ${ }^{27}$ Our results suggest that this is also the case for asthmatic children, at least those with the studied profile.

In conclusion, our results show that the less fit asthmatic children were able to normalise their aerobic fitness with a supervised training programme without clinical complications. Their ability to improve aerobic capacity was not related to clinical and spirometric severity before training. Interestingly, we found a significant association between aerobic improvement and reduction in use of both inhaled and oral steroids. Further research using larger samples is needed to confirm these findings and to assess the duration of the training induced beneficial effects in treatment requirements, the effectiveness of long term aerobic exercise, the response to submaximal protocols and, finally, to determine which profile of childhood asthma is likely to respond to exercise therapy.

This study was partially supported by research grants from CNPq and FAPESP, Brazil. The authors thank Wandiney A F Carvalho, Respiratory Nurse of the Darcy Vargas Children's Hospital (Sao Paulo, Brazil) and Sandra R R Lucas (Federa University of Sao Paulo, Sao Paulo, Brazil) for their technical assistance in performing the exercise challenge and cardiopulmonary exercise tests, respectively. The authors are indebted to Mrs Patricia Chapman (Department of Physiology, St George's Medical School) for her careful revision of the English language.

1 American College of Sports Medicine. Guidelines for graded exercise testing and training. 4th edn. Philadelphia: Lea \& Febiger, 1991.

$2 \mathrm{McF}$ adden ER. Exercise performance in the asthmatic. $A m$ Rev Respir Dis 1984;129:584-7.

3 Cochrane LM, Clark CJ. Benefits and problems of a physical training programme for asthmatic patients. Thorax cal training prograr
4 Garfinkel SK, Kesten S, Chapman KR, et al. Physiologic and nonphysiologic determinants of aerobic fitness in mild to moderate asthma. Am Rev Respir Dis 1992;145:741-5.

5 Ludwick SK, Jones JW, Jones TX, et al. Normalization of cardiopulmonary endurance in severely asthmatic children after bicycle ergometry therapy. F Pediatr 1986;109:446-51.

6 Robinson DM, Egglestone DM, Hill PM, et al. Effects of a physical conditioning programme in asthmatic patients. $N$ $Z$ Med F 1992;937:253-6.

7 Strunk RC, Rubin D, Kelly L, et al. Determination of fitness in children with asthma: use of standardized tests for functional endurance, body fat composition, flexibility, and abdominal strengh. Am f Dis Child 1988;142:940-4.

8 Thio BJ, Nagelkerke AF, Ketel AG, et al. Exercise-induced asthma and cardiovascular fitness in asthmatic children. Thorax 1996;51:207-9.

9 National Heart Lung and Blood Institute. International consensus report on diagnosis and management of asthma. Publication No. 92-3091, NHLBI, 1992.

10 American Thoracic Society. Guidelines for the evaluation of impairment/disability in patients with asthma. Am Rev Respir Dis 1993;147:1056-61.

11 American Thoracic Society. Lung function testing: selection of reference values and interpretative strategies. Am Rev Respir Dis 1991;144:1202-18.

12 Knudson RJ, Lebowitz MD, Holberg CJ, et al. Changes in the normal maximal expiratory flow-volume curve with growth and aging. Am Rev Respir Dis 1983;127:725-34.

13 Godfrey S, Davies CTM, Wozniak E, et al. Cardiorespiratory responses to exercise in normal children. Clin Sci 1971;40:419-31.

14 Cooper DM, Weiler-Ravell D, Whipp BJ et al. Aerobic parameters of exercise as a function of body size during growth in children. $\mathcal{F}$ Appl Physiol 1984;56:628-34.

15 Beaver WL, Wasserman K, Whipp BJ. A new method for detecting the anaerobic threshold by gas exchange. $7 \mathrm{Appl}$ Physiol 1986;60:2020-7.

16 Eggleston PA, Rosenthal RR, Anderson SA, et al. Guidelines for the methodology of exercise challenge testing of asthmatics. F Allergy Clin Immunol 1979;64:642-5.

17 Fink G, Kaye C, Blau H, et al. Assessment of exercise capacity in asthmatic children with various degrees of activity. Pediatr Pulmonol 1993;15:41-3.

18 McArdle WD, Katch FI, Katch FL. Training for anaerobic and aerobic power. In: Exercise physiology. 4th edn. Baltimore: Williams \& Wilkins, 1996: 393-415.

19 Brodal P, Ingjer F, Hermansen L. Cappilary supply of skeletal muscle fibers in untrained and endurance-trained men. Am 7 Physiol 1977;232:H705-12.

20 Hanania NA, Chapman KR, Kesten S. Adverse effects of inhaled corticosteroids. Am f Med 1995;98:196-208.

21 Yiallourds PK, Milner AD, Conway E, et al. Adrenal function and high dose inhaled corticosteroids for asthma. Arch Dis Child 1997;76:405-10.

22 Strunk RC, Mrazek DA, Fukuhara JT, et al. Cardiovascular fitness in children with asthma correlates with psychologic functioning of the child. Pediatrics 1989;84:460-4.

23 Engström I, Fälström K, Karlborg E, et al. Psychological and respiratory physiological effects of a physical exercise programme on boys with severe asthma. Acta Paediatr Scand 1991;80:1058-62.

24 Nery LE, Silva AC, Neder JA, et al. Exercise tolerance and anaerobic threshold (AT) in children with moderate to severe bronchial asthma. Am f Respir Crit Care Med 1994; 149:A786.

25 Bungaard A, Ingemann-Hansen T, Schimdt A, et al. The importance of ventilation in exercise-induced asthma. Allergy 1981;36:385-9.

26 Cooper DM. Rethinking exercise testing in children: a challenge. Am f Respir Crit Care Med 1995;152:1154-7.

27 Santuz P, Baraldi E, Filippone M, et al. Exercise performance in children with asthma: is it different from that of healthy controls? Eur Respir 7 1997;10:1254-60. 